\title{
A Quasistatic Electro-Viscoelastic Contact Problem with Adhesion
}

\author{
Nadhir Chougui ${ }^{1}$ - Salah Drabla ${ }^{1}$
}

Received: 7 December 2013 / Revised: 9 March 2014 / Published online: 22 September 2015

(C) The Author(s) 2015. This article is published with open access at Springerlink.com

\begin{abstract}
The aim of this paper is to study the process of contact with adhesion between a piezoelectric body and an obstacle, the so-called foundation. The material's behavior is assumed to be electro-viscoelastic; the process is quasistatic, the contact is modeled by the Signorini condition. The adhesion process is modeled by a bonding field on the contact surface. We derive a variational formulation for the problem and then we prove the existence of a unique weak solution to the model. The proof is based on a general result on evolution equations with maximal monotone operators and fixed-point arguments.
\end{abstract}

Keywords Electro-viscoelastic material - Signorini's condition · Adhesion · Quasi-variational inequality · Maximal monotone operator · Weak solution · Fixed point

Mathematics Subject Classification 35J87 · 74M10 · 74M15 · $49 \mathrm{~J} 40$

\section{Introduction}

A piezoelectric body is one that produces an electric charge when a mechanical stress is applied (the body is squeezed or stretched). Conversely, a mechanical deformation (the body shrinks or expands) is produced when an electric field is applied. This

\footnotetext{
Communicated by Ahmad Izani MD Ismail.

$\bowtie \quad$ Nadhir Chougui

chouguinadhir@yahoo.fr

Salah Drabla

drabla_s@yahoo.fr

1 Departement of Mathematics, Faculty of Sciences, University of Sétif 1, 19000 Sétif, Algeria
} 
kind of materials appears usually in the industry as switches in radiotronics, electroacoustics, or measuring equipments. Piezoelectric materials for which the mechanical properties are elastic are also called electro-elastic materials, and those for which the mechanical properties are viscoelastic are also called electro-viscoelastic materials. Different models have been developed to describe the interaction between the electrical and mechanical fields( see, e.g., [2,14,16-19,29-31] and the references therein). General models for elastic materials with piezoelectric effect, called electro-elastic materials, can be found in $[2,4,14]$. A static frictional contact problem for electricelastic materials was considered in $[1,15]$, under the assumption that the foundation is insulated. Contact problems involving elasto-piezoelectric materials $[1,15,28]$, viscoelastic piezoelectric materials $[5,25]$ have been studied.

Adhesion may take place between parts of the contacting surfaces. It may be intentional, when surfaces are bonded with glue, or unintentional, as a seizure between very clean surfaces. The adhesive contact is modeled by a bonding field on the contact surface, denoted in this paper by $\beta$; it describes the pointwise fractional density of active bonds on the contact surface, and sometimes referred to as the intensity of adhesion. Following $[10,11]$, the bonding field satisfies the restrictions $0 \leq \beta \leq 1$; when $\beta=1$ at a point of the contact surface, the adhesion is complete and all the bonds are active; when $\beta=0$ all the bonds are inactive, severed, and there is no adhesion; when $0<\beta<1$ the adhesion is partial and only a fraction $\beta$ of the bonds is active. Basic modeling can be found in [10-12]. Analysis of models for adhesive contact can be found in [7,8] and in the monographs [24,27]. An application of the theory of adhesive contact in the medical field of prosthetic limbs was considered in $[22,23]$; there, the importance of the bonding between the bone-implant and the tissue was outlined, since debonding may lead to decrease in the persons ability to use the artificial limb or joint.

In this work we continue in this line of research, where we extend the result established in $[3,20]$ for contact problem described with the Signorini conditions into contact problem described with the Signorini conditions with adhesion where the obstacle is a perfect insulator and the resistance to tangential motion is generated by the glue, in comparison to which the frictional traction can be neglected. Therefore, the tangential contact traction depends only on the bonding field and the tangential displacement.

The paper is structured as follows. In Sect. 2 we present the electro-viscoelastic contact model with adhesion and provide comments on the contact boundary conditions. In Sect. 3 we list the assumptions on the data and derive the variational formulation. In Sect. 4, we present our main existence and uniqueness result, Theorem 4.1, which states the unique weak solvability of the Signorini adhesive contact problem. The proof of the theorem is provided in Sect. 5, where it is carried out in several steps and is based on a general result on evolution equations with maximal monotone operators and fixed-point theorem.

\section{The Model}

We consider a body made of a piezoelectric material which occupies the domain $\Omega \subset \mathbb{R}^{d}(d=2,3)$ with a smooth boundary $\partial \Omega=\Gamma$ and a unit outward normal 
$\nu$. The body is acted upon by body forces of density $f_{0}$ and has volume free electric charges of density $q_{0}$. It is also constrained mechanically and electrically on the boundary. To describe these constraints we assume a partition of $\Gamma$ into three open disjoint parts $\Gamma_{1}, \Gamma_{2}$, and $\Gamma_{3}$, on the one hand, and a partition of $\Gamma_{1} \cup \Gamma_{2}$ into two open parts $\Gamma_{a}$ and $\Gamma_{b}$, on the other hand. We assume that meas $\Gamma_{1}>0$ and meas $\Gamma_{a}>0$; these conditions allow the use of coercivity arguments in the proof of the unique solvability of the model. The body is clamped on $\Gamma_{1}$, and therefore, the displacement field vanishes there. Surface tractions of density $f_{2}$ act on $\Gamma_{2}$. We also assume that the electrical potential vanishes on $\Gamma_{a}$ and a surface electrical charge of density $q_{2}$ is prescribed on $\Gamma_{b}$. On $\Gamma_{3}$ the body is in adhesive contact with an insulator obstacle, the so-called foundation. The contact is frictionless and, since the foundation is assumed to be rigid, we model it with the Signorini condition.

We are interested in the deformation of the body on the time interval $[0 T]$. The process is assumed to be quasistatic, i.e., the inertial effects in the equation of motion are neglected. We denote by $x \in \Omega \cup \Gamma$ and $t \in[0 T]$ the spatial and the time variable, respectively, and, to simplify the notation, we do not indicate in what follows the dependence of various functions on $x$ and $t$. Here and everywhere in this paper, $i$, $j, k, l=1, \ldots, d$, summation over two repeated indices is implied, and the index that follows a comma represents the partial derivative with respect to the corresponding component of $x$. The dot above variable represents the time derivatives.

We denote by $\mathbb{S}^{d}$ the space of second-order symmetric tensors on $\mathbb{R}^{d}(d=2,3)$ and by ".", $\|$.$\| the inner product and the norm on \mathbb{S}^{d}$ and $\mathbb{R}^{d}$, respectively, that is $u . v=u_{i} v_{i},\|v\|=(v . v)^{1 / 2}$ for $u=\left(u_{i}\right), v=\left(v_{i}\right) \in \mathbb{R}^{d}$, and $\sigma \cdot \tau=\sigma_{i j} \tau_{i j}$, $\|\sigma\|=(\sigma . \sigma)^{1 / 2}$ for $\sigma=\left(\sigma_{i j}\right), \tau=\left(\tau_{i j}\right) \in \mathbb{S}^{d}$. We also use the usual notation for the normal components and the tangential parts of vectors and tensors, respectively, given by $v_{v}=v \cdot v, v_{\tau}=v-v_{v} \nu, \sigma_{v}=\sigma_{i j} v_{i} \nu_{j}$, and $\sigma_{\tau}=\sigma v-\sigma_{v} \nu$.

With these assumptions, the classical model for the process is the following.

Problem $1(\mathcal{P})$. Find a displacement field $u: \Omega \times[0, T] \rightarrow \mathbb{R}^{d}$, a stress field $\sigma: \Omega \times[0, T] \rightarrow \mathbb{S}^{d}$, an electric potential $\varphi: \Omega \times[0, T] \rightarrow \mathbb{R}$, an electric displacement field $D: \Omega \times[0, T] \rightarrow \mathbb{R}^{d}$, and a bonding field $\beta: \Omega \times[0, T] \rightarrow \mathbb{R}$ such that

$$
\begin{aligned}
& \sigma=\mathcal{A} \varepsilon(\dot{u})+\mathcal{F} \varepsilon(u)-\mathcal{E}^{*} \mathbf{E}(\varphi) \quad \text { in } \quad \Omega \times(0, T), \\
& D=\mathcal{B} \mathbf{E}(\varphi)+\mathcal{E} \varepsilon(u) \quad \text { in } \Omega \times(0, T) \text {, } \\
& \operatorname{Div} \sigma+f_{0}=0 \quad \text { in } \Omega \times(0, T) \text {, } \\
& \operatorname{div} D=q_{0} \quad \text { in } \Omega \times(0, T) \text {, } \\
& u=0 \\
& \sigma v=f_{2} \\
& \text { on } \Gamma_{1} \times(0, T) \text {, } \\
& \text { on } \quad \Gamma_{2} \times(0, T) \\
& \left\{\begin{array}{l}
u_{v} \leq 0, \\
\sigma_{v}-\gamma_{v} \beta^{2} R_{v}\left(u_{v}\right) \leq 0, \\
\left(\sigma_{v}-\gamma_{v} \beta^{2} R_{v}\left(u_{v}\right)\right) u_{v}=0
\end{array}\right. \\
& \text { on } \quad \Gamma_{3} \times(0, T) \text {, } \\
& -\sigma_{\tau}=p_{\tau}(\beta) R_{\tau}\left(u_{\tau}\right) \\
& \text { on } \quad \Gamma_{3} \times(0, T) \text {, } \\
& \dot{\beta}(t)=-\left(\gamma_{\nu} \beta(t) R_{v}\left(u_{v}(t)\right)^{2}-\varepsilon_{a}\right)_{+} \quad \text { on } \quad \Gamma_{3} \times(0, T) \text {, }
\end{aligned}
$$




$$
\begin{array}{lll}
\varphi=0 & \text { on } & \Gamma_{a} \times(0, T), \\
D . v=q_{2} & \text { on } & \Gamma_{b} \times(0, T), \\
D . v=0 & \text { on } & \Gamma_{3} \times(0, T), \\
u(0)=u_{0} & \text { in } & \Omega, \\
\beta(0)=\beta_{0} & \text { on } & \Gamma_{3} .
\end{array}
$$

We now provide some comments on equations and conditions (2.1)-(2.14).

First, equations (2.1) and (2.2) represent the electro-viscoelastic constitutive law in which $\sigma=\left(\sigma_{i j}\right)$ is the stress tensor, $\varepsilon(u)=\left(\varepsilon_{i j}(u)\right)$ denotes the linearized strain tensor, $\mathbf{E}(\varphi)=-\nabla \varphi$ is the electric field, $\mathcal{A}$ and $\mathcal{F}$ are the viscosity and elasticity operators, respectively, $\mathcal{E}=\left(e_{i j k}\right)$ represents the third-order piezoelectric tensor, $\mathcal{E}^{*}=$ $\left(e_{i j k}^{*}\right)$, where $e_{i j k}^{*}=e_{k i j}$, is its transpose, $\mathcal{B}=\left(\mathcal{B}_{i j}\right)$ denotes the electric permittivity tensor, and $D=\left(D_{1}, \ldots, D_{d}\right)$ is the electric displacement vector. Details on the constitutive equations of the form (2.1) and (2.2) can be found, for instance, in [1,2, $13,21]$ and the references therein.

Next, Eqs. (2.3) and (2.4) are the equilibrium equations for the stress and electric displacement fields, respectively, in which Div" and "div" denote the divergence operators for tensor and vector valued functions, respectively.

Conditions (2.5) and (2.6) are the displacement and traction boundary conditions, whereas (2.10) and (2.11) represent the electric boundary conditions. Note that we need to impose assumption (2.12) for physical reasons. Indeed, this condition models the case when the obstacle is a perfect insulator and was used in $[1,9,15,25,26]$. The evolution of the bonding field is governed by the differential Eq. (2.9) with given positive parameters $\gamma_{v}$ and $\varepsilon_{a}$ where $r_{+}=\max \{0, r\}$.

Condition (2.7) represents the Signorini contact condition with adhesion, where $u_{v}$ is the normal displacement, $\sigma_{v}$ represents the normal stress, $\gamma_{v}$ denotes a given adhesion coefficient, and $R_{v}$ is the truncation operator defined by

$$
R_{v}(s)= \begin{cases}-L & \text { if } s<-L \\ -s & \text { if }-L \leq s \leq 0 \\ 0 & \text { if } s>0\end{cases}
$$

Here $L>0$ is the characteristic length of the bond, beyond which it does not offer any additional traction (see [27]).

We assume that the resistance to tangential motion is generated only by the glue, and is assumed to depend on the adhesion field and on the tangential displacement, but, again, only up to the bond length $L$ (see (2.8)), where the truncation operator $R_{\tau}$ is defined by

$$
R_{\tau}(v)= \begin{cases}v & \text { if }\|v\| \leq L \\ L \frac{v}{\|v\|} & \text { if }\|v\|>L\end{cases}
$$

Then, $p_{\tau}(\beta)$ acts as the stiffness or spring constant, increasing with $(\beta)$, and the traction is in the direction opposite to the displacement. The maximal modulus of the tangential traction is $p_{\tau}(1) L$. 
Finally, (2.13) and (2.14) represent the initial conditions in which $u_{0}$ and $\beta_{0}$ are the prescribed initial displacement and bonding fields, respectively.

\section{Variational Formulation and Preliminaries}

In this section, we list the assumptions on the data and derive a variational formulation for the contact problem. To this end we need to introduce some notation and preliminaries.

Everywhere below, we use the classical notation for $L^{p}$ and Sobolev spaces associated to $\Omega$ and $\Gamma$. Moreover, we use the notation $L^{2}(\Omega)^{d}, H^{1}(\Omega)^{d}, \mathcal{H}$, and $\mathcal{H}_{1}$ for the following spaces

$$
\begin{aligned}
& L^{2}(\Omega)^{d}=\left\{v=\left(v_{i}\right) \mid v_{i} \in L^{2}(\Omega)\right\}, \quad H^{1}(\Omega)^{d}=\left\{v=\left(v_{i}\right) \mid v_{i} \in H^{1}(\Omega)\right\}, \\
& \mathcal{H}=\left\{\tau=\left(\tau_{i j}\right) \mid \tau_{i j}=\tau_{j i} \in L^{2}(\Omega)\right\}, \quad \mathcal{H}_{1}=\left\{\tau \in \mathcal{H} \mid \tau_{i j, j} \in L^{2}(\Omega)\right\} .
\end{aligned}
$$

The spaces $L^{2}(\Omega)^{d}, H^{1}(\Omega)^{d}, \mathcal{H}$, and $\mathcal{H}_{1}$ are real Hilbert spaces endowed with the canonical inner products given by

$$
\begin{aligned}
& (u, v)_{L^{2}(\Omega)^{d}}=\int_{\Omega} u \cdot v d x, \quad(u, v)_{H^{1}(\Omega)^{d}}=\int_{\Omega} u \cdot v d x+\int_{\Omega} \nabla u \cdot \nabla v d x, \\
& (\sigma, \tau)_{\mathcal{H}}=\int_{\Omega} \sigma \cdot \tau d x, \quad(\sigma, \tau)_{\mathcal{H}_{1}}=\int_{\Omega} \sigma \cdot \tau d x+\int_{\Omega} \operatorname{Div} \sigma \cdot \operatorname{Div} \tau d x,
\end{aligned}
$$

and the associated norms $\|\cdot\|_{L^{2}(\Omega)^{d}},\|\cdot\|_{H^{1}(\Omega)^{d}},\|\cdot\|_{\mathcal{H}}$, and $\|\cdot\|_{\mathcal{H}_{1}}$, respectively. Here and below we use the notation

$$
\begin{aligned}
& \nabla v=\left(v_{i, j}\right), \quad \varepsilon(v)=\left(\varepsilon_{i j}(v)\right), \quad \varepsilon_{i j}(v)=\frac{1}{2}\left(v_{i, j}+v_{j, i}\right) \quad \forall v \in H^{1}(\Omega)^{d}, \\
& \operatorname{Div} \tau=\left(\tau_{i j, j}\right) \quad \forall \tau \in \mathcal{H}_{1} .
\end{aligned}
$$

For every element $v \in H^{1}(\Omega)^{d}$ we also write $v$ for the trace of $v$ on $\Gamma$ and we denote by $v_{v}$ and $v_{\tau}$ the normal and tangential components of $v$ on $\Gamma$.

We now list the assumptions on the problem's data. The viscosity operator $\mathcal{A}$ and the elasticity operator $\mathcal{F}$ are assumed to satisfy the conditions

(a) $\mathcal{A}: \Omega \times \mathbb{S}^{d} \longrightarrow \mathbb{S}^{d}$.

(b) $\mathcal{A}(x, \tau)=\left(a_{i j k l}(x) \tau_{k l}\right) \forall \tau \in S^{d}$ a.e. $x \in \Omega$.

(c) $a_{i j k l}=a_{k l i j}=a_{j i k l} \in L^{\infty}(\Omega)$.

(d) there exists $m_{\mathcal{A}}>0$ such that $a_{i j k l} \tau_{i j} \tau_{k l} \geq m_{\mathcal{A}}\|\tau\|^{2}, \forall \tau=\left(\tau_{i j}\right) \in \mathbb{S}^{d}$, a.e. $x \in \Omega$.

(a) $\mathcal{F}: \Omega \times \mathbb{S}^{d} \rightarrow \mathbb{S}^{d}$.

(b) There exists $L_{\mathcal{F}}>0$ such that $\left\|\mathcal{F}\left(x, \tau_{1}\right)-\mathcal{F}\left(x, \tau_{2}\right)\right\| \leq L_{\mathcal{F}}\left\|\tau_{1}-\tau_{2}\right\|$ $\forall \tau_{1}, \tau_{2} \in \mathbb{S}^{d}$, a.e. $\mathbf{x} \in \Omega$.

(c) The mapping $x \mapsto \mathcal{F}(x, \tau)$ is measurable on $\Omega$, for each $\tau \in \mathbb{S}^{d}$.

(d) The mapping $x \mapsto \mathcal{F}(x, \mathbf{0})$ belongs to $\mathcal{H}$. 
The piezoelectric tensor $\mathcal{E}$ and the electric permittivity tensor $\mathcal{B}$ satisfy

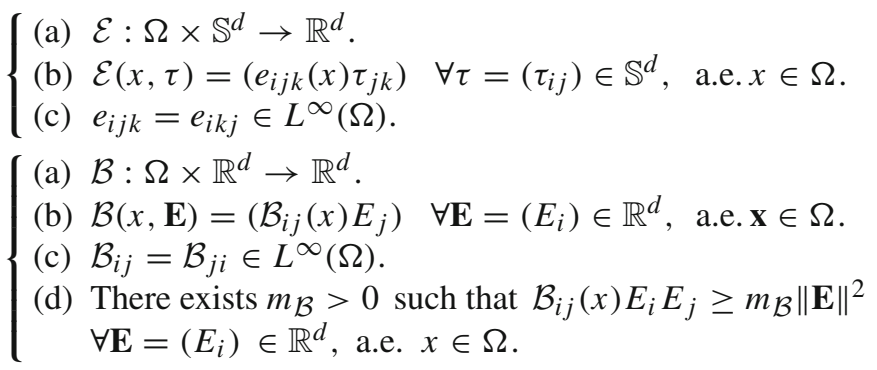

As in [8] we assume that the tangential contact function satisfies

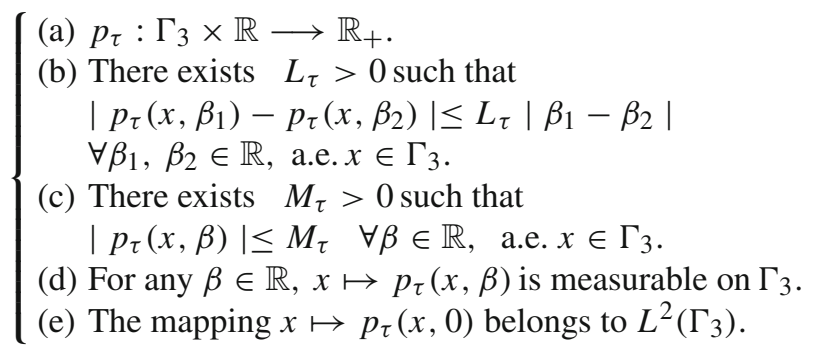

The forces, tractions, volume, and surface-free charge densities satisfy

$$
\begin{aligned}
& f_{0} \in W^{1,1}\left(0, T ; L^{2}(\Omega)^{d}\right), \quad f_{2} \in W^{1,1}\left(0, T ; L^{2}\left(\Gamma_{2}\right)^{d}\right), \\
& q_{0} \in W^{1,1}\left(0, T ; L^{2}(\Omega)\right), \quad q_{2} \in W^{1,1}\left(0, T ; L^{2}\left(\Gamma_{b}\right)\right) .
\end{aligned}
$$

The adhesion coefficient $\gamma_{v}$ and the limit bound $\varepsilon_{a}$ satisfy the conditions

$$
\gamma_{\nu} \in L^{\infty}\left(\Gamma_{3}\right), \quad \epsilon_{a} \in L^{2}\left(\Gamma_{3}\right), \quad \gamma_{\nu}, \gamma_{\tau}, \epsilon_{a} \geq 0 \text { a.e. on } \Gamma_{3} .
$$

Also, we assume that the initial bonding field satisfies

$$
\beta_{0} \in L^{2}\left(\Gamma_{3}\right), \quad 0 \leq \beta_{0} \leq 1 \text { a.e. on } \Gamma_{3} \text {. }
$$

Moreover, the tensor $\mathcal{E}$ and its transpose $\mathcal{E}^{*}$ satisfy the equality

$$
\mathcal{E} \sigma . v=\sigma \cdot \mathcal{E}^{*} v \quad \forall \sigma \in \mathbb{S}^{d}, v \in \mathbb{R}^{d}
$$

Let now consider the closed subspace of $H^{1}(\Omega)^{d}$ defined by

$$
V=\left\{v \in H^{1}(\Omega)^{d} \mid v=0 \text { on } \Gamma_{1}\right\} .
$$

Since meas $\left(\Gamma_{1}\right)>0$ and the viscosity tensor satisfies assumption (3.1), it follows that $V$ is a real Hilbert space endowed with the inner product 


$$
(u, v)_{V}=(\mathcal{A} \varepsilon(u), \varepsilon(v))_{\mathcal{H}},
$$

and let $\|\cdot\|_{V}$ be the associated norm.

We also introduce the following spaces

$W=\left\{\psi \in H^{1}(\Omega) \mid \psi=0\right.$ on $\left.\Gamma_{a}\right\}, \mathcal{W}=\left\{D=\left(D_{i}\right) \mid D_{i} \in L^{2}(\Omega), \operatorname{div} D \in L^{2}(\Omega)\right\}$.

Since meas $\left(\Gamma_{a}\right)>0$ it is well known that $W$ is a real Hilbert space endowed with the inner product

$$
(\varphi, \psi)_{W}=(\nabla \varphi, \nabla \psi)_{L^{2}(\Omega)^{d}},
$$

and the associated norm $\|\cdot\|_{W}$. Also we have the following Friedrichs-Poincaré inequality

$$
\|\nabla \psi\|_{L^{2}(\Omega)^{d}} \geq c_{F}\|\psi\|_{H^{1}(\Omega)} \quad \forall \psi \in W,
$$

where $c_{F}>0$ is a constant which depends only on $\Omega$ and $\Gamma_{a}$. The space $\mathcal{W}$ is a real Hilbert space endowed with the inner product

$$
(D, \mathbf{E})_{\mathcal{W}}=\int_{\Omega} D \cdot \mathbf{E} d x+\int_{\Omega} \operatorname{div} D \cdot \operatorname{div} \mathbf{E} d x
$$

and the associated norm $\|\cdot\|_{\mathcal{W}}$. Moreover, by the Sobolev trace theorem, there exist two positive constants $c_{0}$ and $\tilde{c}_{0}$ such that

$$
\|v\|_{L^{2}\left(\Gamma_{3}\right)^{d}} \leq c_{0}\|v\|_{V} \quad \forall v \in V,\|\psi\|_{L^{2}\left(\Gamma_{3}\right)} \leq \tilde{c}_{0}\|\psi\|_{W} \quad \forall \psi \in W .
$$

Next, we define the two mappings $f:[0 T] \longrightarrow V$ and $q:[0 T] \longrightarrow W$, respectively, by

$$
\begin{aligned}
& (f(t), v)_{V}=\int_{\Omega} f_{0}(t) \cdot v d x+\int_{\Gamma_{2}} f_{2}(t) \cdot v d a \\
& (q(t), \psi)_{W}=\int_{\Omega} q_{0}(t) \psi d x-\int_{\Gamma_{b}} q_{2}(t) \psi d a
\end{aligned}
$$

for all $v \in V, \psi \in W$, and $t \in[0, T]$. We note that the definitions of $f$ and $q$ are based on the Riesz representation theorem. Moreover, it follows from assumptions (3.6) and (3.7) that

$$
\begin{aligned}
& f \in W^{1,1}(0, T ; V), \\
& q \in W^{1,1}(0, T ; W) .
\end{aligned}
$$

For Signorini problem, we use the convex subset of admissible displacements fields given by

$$
U_{a d}=\left\{v \in V / v_{v} \leq 0 \text { on } \Gamma_{3}\right\},
$$

and we make the regularity assumption

$$
u_{0} \in U_{a d}
$$


on the initial data. Also, we introduce the set

$$
\mathcal{Q}=\left\{\beta \in L^{\infty}\left(0, T ; L^{2}\left(\Gamma_{3}\right)\right) \mid 0 \leq \beta(t) \leq 1 \forall t \in[0, T] \text {, a.e. on } \Gamma_{3}\right\}
$$

Next, we define the functional $j: L^{2}\left(\Gamma_{3}\right) \times V \times V \longrightarrow \mathbb{R}$ by

$$
j(\beta, u, v)=\int_{\Gamma_{3}}-\gamma_{\nu} \beta^{2} R_{v}\left(u_{\nu}\right) v_{\nu} d a+\int_{\Gamma_{3}} p_{\tau}(\beta) R_{\tau}\left(u_{\tau}\right) \cdot v_{\tau} d a .
$$

It follows from assumptions (3.5)-(3.8) that the integrals in (3.14), (3.15), and (3.19) are well defined.

Using a standard procedure based on Green's formulas and equalities (3.14), (3.15), (3.19), it is easy to see that if $(u, \sigma, \varphi, \beta, D)$ are sufficiently regular functions which satisfy (2.3)-(2.12) then

$$
\begin{gathered}
u(t) \in U_{a d},(\sigma(t), \varepsilon(v)-\varepsilon(u(t)))_{\mathcal{H}}+j\left(\beta(t), u(t), v-u(t) \geq(f(t), v-u(t))_{V},\right. \\
(D(t), \nabla \psi)_{L^{2}(\Omega)^{d}}+(q(t), \psi)_{W}=0,
\end{gathered}
$$

for all $v \in U_{a d}, \psi \in W$ and $t \in[0, T]$. We substitute (2.1) in (3.20), (2.2) in (3.21), keeping in mind that $\mathbf{E}(\varphi)=-\nabla \varphi$ and use the initial condition (2.13) to derive the following variational formulation of $\operatorname{Problem}(\mathcal{P})$.

Problem $2\left(\mathcal{P}^{V}\right)$. Find a displacement field $u:[0, T] \longrightarrow V$, an electric potential field $\varphi:[0, T] \rightarrow W$, and a bonding field $\beta:[0, T] \rightarrow L^{2}\left(\Gamma_{3}\right)$ such that

$$
\begin{aligned}
& u(t) \in U_{a d},(\mathcal{A} \varepsilon(\dot{u}(t)), \varepsilon(v)-\varepsilon(u(t)))_{\mathcal{H}}+(\mathcal{F} \varepsilon(u(t)), \varepsilon(v)-\varepsilon(u(t)))_{\mathcal{H}} \\
& +\left(\mathcal{E}^{*} \nabla \varphi(t), \varepsilon(v)-\varepsilon(u(t))\right)_{\mathcal{H}}+j(\beta(t), u(t), v-u(t)) \\
& \geq(f(t), v-u(t))_{V} \quad \forall v \in U_{a d} \text {, a.e. } t \in(0 T), \\
& (\mathcal{B} \nabla \varphi(t), \nabla \psi)_{L^{2}(\Omega)^{d}}-(\mathcal{E} \varepsilon(u(t)), \nabla \psi)_{L^{2}(\Omega)^{d}}=(q(t), \psi)_{W} \\
& \times \forall \psi \in W, \forall t \in\left[\begin{array}{ll}
0 & T
\end{array}\right], \\
& \dot{\beta}(t)=-\left(\gamma_{v} \beta(t) R_{v}\left(u_{v}(t)\right)^{2}-\varepsilon_{a}\right)_{+} \text {. a.e. } t \in(0 T), \\
& u(0)=u_{0}, \\
& \beta(0)=\beta_{0} \text {. }
\end{aligned}
$$

\section{Existence and Uniqueness Result}

Our main existence and uniqueness result is the following.

Theorem 4.1 Assume that (3.1)-(3.9) and (3.18) hold. Then, there exists a unique solution $(u, \varphi, \beta)$ to Problem $\left(\mathcal{P}^{V}\right)$. Moreover, the solution satisfies

$$
\begin{aligned}
& \boldsymbol{u} \in W^{1, \infty}(0, T ; V), \\
& \varphi \in W^{1, \infty}(0, T ; W),
\end{aligned}
$$




$$
\beta \in W^{1, \infty}\left(0, T ; L^{2}\left(\Gamma_{3}\right)\right) \cap \mathcal{Q}
$$

A "quintuple; of functions $(u, \sigma, \varphi, D, \beta)$ which satisfies (2.1), (2.2) and (3.22)(3.26) is called a weak solution of the contact Problem (P). We conclude by Theorem 4.1 that, under the assumptions (3.1)-(3.9) and (3.18), there exists a unique weak solution of Problem $(\mathcal{P})$.

To precise the regularity of the weak solution we note that the constitutive relations (2.1) and (2.2), the assumptions (3.1)-(3.4) and the regularities (4.1), (4.2) imply that $\sigma \in L^{\infty}(0, T ; \mathcal{H}), \boldsymbol{D} \in W^{1, \infty}\left(0, T ; L^{2}(\Omega)^{d}\right)$. By taking $v=u(t) \pm \xi$, where $\xi \in C_{0}^{\infty}(\Omega)^{d}$, in (3.20) and $\psi \in C_{0}^{\infty}(\Omega)$ in (3.21) and using the notation (3.14), (3.15), (3.19) we find

$$
\operatorname{Div} \sigma(t)+f_{0}(t)=0, \operatorname{div} D(t)=q_{0}(t),
$$

for all $t \in[0, T]$. It follows now from the regularities (3.6), (3.7) that Div $\sigma \in$ $L^{\infty}\left(0, T ; L^{2}(\Omega)^{d}\right)$ and div $D \in W^{1, \infty}\left(0, T ; L^{2}(\Omega)\right)$, which shows that

$$
\begin{aligned}
& \sigma \in L^{\infty}\left(0, T ; \mathcal{H}_{1}\right), \\
& D \in W^{1, \infty}(0, T ; \mathcal{W}) .
\end{aligned}
$$

We conclude that the weak solution $(u, \sigma, \varphi, D, \beta)$ of the piezoelectric contact problem $(\mathcal{P})$ has the regularity $(4.1)-(4.5)$.

The proof of Theorem 4.1 will be carried out in several steps and is based on the following abstract result.

Let $X$ be a real Hilbert space with the inner product $(\cdot, \cdot)_{X}$ and the associated norm $\|\cdot\|_{X}$, and let $A: D(A) \subset X \longrightarrow 2^{X}$ be a multivalued operator, where $D(A)$ is the domain of $A$ given by

$$
D(A)=\{x \in X: A x \neq \varnothing\},
$$

and $2^{X}$ represents the set of the subsets of $X$. The graph of $A$ denoted by $\operatorname{Gr}(A)$ is given by

$$
\operatorname{Gr}(A)=\{(x, y) \in X \times X: y \in A x\} .
$$

The operator $A: X \longrightarrow 2^{X}$ is called

(i) monotone if

$$
\forall\left(x_{1}, y_{1}\right) \in \operatorname{Gr}(A), \forall\left(x_{2}, y_{2}\right) \in \operatorname{Gr}(A):\left(y_{1}-y_{2}, x_{1}-x_{2}\right)_{X} \geq 0 .
$$

(ii) maximal monotone if $A$ is monotone and there is no monotone

operator $B: X \longrightarrow 2^{X}$ such that $\operatorname{Gr}(A)$ is a proper subset of $\operatorname{Gr}(B)$,

which is equivalent to the following implication

$\left[\left(y_{1}-y_{2}, x_{1}-x_{2}\right)_{X} \geq 0, \forall\left(x_{1}, y_{1}\right) \in \operatorname{Gr}(A)\right] \Rightarrow\left(x_{2}, y_{2}\right) \in \operatorname{Gr}(A)$.

For a function $\phi: X \longrightarrow]-\infty,+\infty]$ we use the notation $D(\phi)$ and $\partial \phi$ for the effective domain and the subdifferential of $\phi$, i.e.,

$$
D(\phi)=\{u \in X: \phi(u)<\infty\},
$$




$$
\partial \phi(u)=\left\{f \in X: \phi(v)-\phi(u) \geq(f, v-u)_{X} \quad \forall v \in X\right\}, \forall u \in X .
$$

Finally, let $\left.\phi_{K}: X \rightarrow\right]-\infty,+\infty$ ] denote the indicator function of the set $K$, i.e.,

$$
\phi_{K}(v)= \begin{cases}0 & \text { if } v \in K \\ \infty & \text { if } v \notin K\end{cases}
$$

It can be shown that the subdifferential of the indicator function $\partial \phi_{K}: X \longrightarrow 2^{X}$ of a closed convex $K$ of the space $X$ is a maximal monotone operator. We can also show that the sum of a maximal monotone operator and a single-valued monotone Lipschitz continuous operator is a maximal monotone operator.

Finally, we use the usual notation for the Lebesgue spaces $L^{p}(0, T ; X)$ and Sobolev spaces $W^{k, p}(0, T ; X)$ where $1 \leq p \leq \infty$ and $k \in \mathbb{N}$. We will need the following result for existence and uniqueness proofs.

Theorem 4.2 Let $X$ be a real Hilbert space and let $A: D(A) \subset X \longrightarrow 2^{X}$ be a multivalued operator such that the operator $A+\omega I_{X}$ is maximal monotone for some real $\omega$. Then, for every $f \in W^{1,1}(0, T ; X)$ and $u_{0} \in D(A)$, there exists a unique function $\boldsymbol{u} \in W^{1, \infty}(0, T ; X)$ which satisfies

$$
\begin{aligned}
& \dot{u}(t)+A u(t) \ni f(t) \text { a.e. } t \in(0 T), \\
& u(0)=u_{0} .
\end{aligned}
$$

A proof of Theorem 4.2 may be found in ([6], p. 32). Here and below $I_{X}$ is the identity map on $X$.

\section{Proof of Theorem 4.1}

We assume in the following that the conditions of Theorem 4.1 hold and below we denote by $c$ a generic positive constant which is independent of time and whose value may change from place to place.

By the Riesz representation theorem we can define the following operators $\mathcal{G}$ : $W \longrightarrow W$ and $\mathcal{R}: V \longrightarrow W$, respectively, by

$$
\begin{array}{ll}
(\mathcal{G} \varphi, \psi)_{W}=(\mathcal{B} \nabla \varphi, \nabla \psi)_{L^{2}(\Omega)^{d}} & \forall \varphi, \psi \in W, \\
(\mathcal{R} v, \varphi)_{W}=(\mathcal{E} \varepsilon(v), \nabla \varphi)_{L^{2}(\Omega)^{d}} & \forall \varphi \in W, v \in V .
\end{array}
$$

We can show that $\mathcal{G}$ is a linearly continuous symmetric positive definite operator. Therefore, $\mathcal{G}$ is an invertible operator on $W$. We can also prove that $\mathcal{R}$ is a linear continuous operator on $V$. Let $\mathcal{R}^{*}$ the adjoint of $\mathcal{R}$. Thus, from (3.10) we can write

$$
\left(\mathcal{R}^{*} \varphi, v\right)_{V}=\left(\mathcal{E}^{*} \nabla \varphi, \varepsilon(v)\right)_{\mathcal{H}} \quad \forall \varphi \in W, v \in V
$$

Let $t \in[0 T]$. By introducing (5.1), (5.2) in (3.23) we get

$$
(\mathcal{G} \varphi(t), \psi)_{W}=(\mathcal{R} u(t), \psi)_{W}+(q(t), \psi)_{W} \forall \psi \in W,
$$


where we obtain

$$
\mathcal{G} \varphi(t)=\mathcal{R} u(t)+q(t),
$$

for all $t \in[0, T]$. On the other hand, $\mathcal{G}$ is invertible where the previous equality gives us

$$
\varphi(t)=\mathcal{G}^{-1} \mathcal{R} u(t)+\mathcal{G}^{-1} q(t) .
$$

Now, using (5.3), (5.5), and (3.22) we obtain

$$
\begin{aligned}
& u(t) \in U_{a d},(\mathcal{A} \varepsilon(\dot{u}(t)), \varepsilon(v)-\varepsilon(u(t)))_{\mathcal{H}}+(\mathcal{F} \varepsilon(u(t)), \varepsilon(v)-\varepsilon(u(t)))_{\mathcal{H}} \\
& \quad+\left(\mathcal{R}^{*} \mathcal{G}^{-1} \mathcal{R} u(t), v-u(t)\right)_{V}+j(\beta(t), u(t), v-u(t))_{V} \\
& \geq\left(f(t)-\mathcal{R}^{*} \mathcal{G}^{-1} q(t), v-u(t)\right)_{V} \quad \forall v \in U_{a d} \text { a.e. } t \in(0 T) .
\end{aligned}
$$

Let $\eta \in W^{1, \infty}(0, T ; V)$ be given. In the first step we prove the following existence and uniqueness result for the displacement field.

Lemma 5.1 There exists a unique function $u_{\eta} \in W^{1, \infty}(0, T ; V)$ such that

$$
\begin{aligned}
& u_{\eta}(t) \in U_{a d},\left(\mathcal{A} \varepsilon\left(\dot{u}_{\eta}(t)\right), \varepsilon(v)-\varepsilon\left(u_{\eta}(t)\right)\right)_{\mathcal{H}}+\left(\mathcal{F} \varepsilon\left(u_{\eta}(t)\right), \varepsilon(v)-\varepsilon\left(u_{\eta}(t)\right)\right)_{\mathcal{H}} \\
& \quad+\left(\mathcal{R}^{*} \mathcal{G}^{-1} \mathcal{R} u_{\eta}(t), v-u_{\eta}(t)\right)_{V}+\left(\eta(t), v-u_{\eta}(t)\right)_{V} \\
& \quad \geq\left(f(t)-\mathcal{R}^{*} \mathcal{G}^{-1} q(t), v-u_{\eta}(t)\right)_{V} \quad \forall v \in U_{a d} \text { a.e. } t \in(0 T) \\
& u_{\eta}(0)=u_{0}
\end{aligned}
$$

Proof Let now the operator $L: V \rightarrow V$ defined by

$$
L(v)=\mathcal{R}^{*} \mathcal{G}^{-1} \mathcal{R}(v), \forall v \in V .
$$

Using the properties of the operators $\mathcal{G}, \mathcal{R}$, and $\mathcal{R}^{*}$ we deduce that $L$ is a continuous linear operator on $V$. Thus we have

$$
\left\|L u_{1}-L u_{2}\right\|_{V} \leq\|L\|\left\|u_{1}-u_{2}\right\|_{V} \quad \forall u_{1}, u_{2} \in V
$$

By the Riesz representation theorem we can define an operator $\mathbf{G}: V \longrightarrow V$ by

$$
(\mathbf{G} u, v)_{V}=(\mathcal{F} \varepsilon(u), \varepsilon(v))_{\mathcal{H}}+(L u, v)_{V} \quad \forall u, v \in V .
$$

Now, taking into account (3.1), (3.2), (3.11) and (5.11) it follows

$$
\left\|\mathbf{G} u_{1}-\mathbf{G} u_{2}\right\|_{V} \leq\left(\frac{L_{\mathcal{F}}}{m_{\mathcal{A}}}+\|L\|\right)\left\|u_{1}-u_{2}\right\|_{V} \quad \forall u_{1}, u_{2} \in V,
$$

that is, $\mathbf{G}$ is a Lipschitz continuous operator. Moreover, the operator

$$
\mathbf{G}+\left(\frac{L_{\mathcal{F}}}{m_{\mathcal{A}}}+\|L\|\right) I_{V}: V \rightarrow V
$$

is a monotone Lipschitz continuous operator on $V$. 
Let the function $\mathbf{f}:[0 T] \longrightarrow V$ given by

$$
\mathbf{f}(t)=f(t)-\mathcal{R}^{*} \mathcal{G}^{-1} q(t)-\eta(t), \forall t \in[0 T]
$$

Keeping in mind that $\eta \in W^{1, \infty}(0, T ; V)$, using (3.16), (3.17) and the fact that $\mathcal{R}^{*} \mathcal{G}^{-1}$ is linearly continuous, it follows from (5.13) that

$$
\mathbf{f} \in W^{1,1}(0, T ; V) \text {. }
$$

Let $\left.\left.\phi_{U_{a d}}: V \rightarrow\right]-\infty,+\infty\right]$ denote the indicator function of the set $U_{a d}$ and let $\partial \phi_{U_{a d}}$ be the subdifferential of $\phi_{U_{a d}}$. Since $U_{a d}$ is a nonempty, convex, closed part of $V$, it follows that $\partial \phi_{U_{a d}}$ is a maximal monotone operator on $V$ and $D\left(\partial \phi_{U_{a d}}\right)=U_{a d}$. Moreover, the sum

$$
\partial \phi_{U_{a d}}+\mathbf{G}+\left(\frac{L_{\mathcal{F}}}{m_{\mathcal{A}}}+\|L\|\right) I_{V}: U_{a d} \subset V \rightarrow 2^{V}
$$

is a maximal monotone operator. Thus, conditions (3.18) and (5.14) allow us to apply Theorem 4.2 with $X=V, A=\partial \phi_{U_{a d}}+\mathbf{G}: D(A)=U_{a d} \subset V \rightarrow 2^{V}$, and $\omega=\frac{L_{\mathcal{F}}}{m_{\mathcal{A}}}+\|L\|$. We deduce that there exists a unique element $u_{\eta} \in W^{1, \infty}(0, T ; V)$ such that

$$
\begin{aligned}
& \dot{u}_{\eta}(t)+\partial \phi_{U_{a d}}\left(u_{\eta}(t)\right)+\mathbf{G} u_{\eta}(t) \ni \mathbf{f}(t) \text { a.e. } t \in(0 T), \\
& u_{\eta}(0)=u_{0} .
\end{aligned}
$$

Since for any elements $u, g \in V$, the following equivalence holds

$$
g \in \partial \phi_{U_{a d}}(u) \Leftrightarrow u \in U_{a d},(g, v-u)_{V} \leq 0 \forall v \in U_{a d},
$$

the differential inclusion (5.15) is equivalent to the following variational inequality

$$
\begin{aligned}
& u_{\eta}(t) \in U_{a d},\left(\dot{u}_{\eta}(t), v-u_{\eta}(t)\right)_{V}+\left(\mathbf{G} u_{\eta}(t), v-u_{\eta}(t)\right)_{V} \\
& \geq\left(\mathbf{f}(t), v-u_{\eta}(t)\right)_{V} \forall v \in U_{a d} \text { a.e. } t \in(0 T) .
\end{aligned}
$$

We use now (5.17), (5.11), (3.11) to see that $u_{\eta}$ satisfies the following inequality

$$
\begin{aligned}
& u_{\eta}(t) \in U_{a d},\left(\mathcal{A} \varepsilon\left(\dot{u}_{\eta}(t)\right), \varepsilon(v)-\varepsilon\left(u_{\eta}(t)\right)\right)_{\mathcal{H}}+\left(\mathcal{F} \varepsilon\left(u_{\eta}(t)\right), \varepsilon(v)-\varepsilon\left(u_{\eta}(t)\right)\right)_{\mathcal{H}} \\
& \quad+\left(L u_{\eta}(t), v-u_{\eta}(t)\right)_{V} \geq\left(\mathbf{f}(t), v-u_{\eta}(t)\right)_{V} \forall v \in U_{a d} \text { a.e. } t \in(0 T) .
\end{aligned}
$$

It follows now from (5.18), (5.13), (5.9), and (5.16) that $u_{\eta}$ satisfies (5.7) and (5.8), which concludes the proof of Lemma 5.1.

In the second step we use the displacement field $u_{\eta}$ obtained in Lemma 5.1 to obtain the following existence and uniqueness result for the electric potential field. 
Lemma 5.2 There exists a unique function $\varphi_{\eta} \in W^{1, \infty}(0, T ; W)$ such that

$$
\begin{aligned}
& \left(\mathcal{B} \nabla \varphi_{\eta}(t), \nabla \psi\right)_{L^{2}(\Omega)^{d}}-\left(\mathcal{E} \varepsilon\left(u_{\eta}(t)\right), \nabla \psi\right)_{L^{2}(\Omega)^{d}}=(q(t), \psi)_{W} \\
& \forall \psi \in W, \forall t \in[0 T]
\end{aligned}
$$

Proof Let $u_{\eta} \in W^{1, \infty}(0, T ; V)$ be the function defined in Lemma 5.1. Clearly, equality (5.19) holds from (5.4), (5.2), and (5.1). Moreover, since $u_{\eta} \in W^{1, \infty}(0, T ; V)$ it follows from (5.5), (3.17) that $\varphi_{\eta} \in W^{1, \infty}(0, T ; W)$. Now, using (5.5) we deduce that the uniqueness of $\varphi_{\eta}$ follows from the uniqueness of the function $u_{\eta}$.

In the third step, we use again the displacement field $u_{\eta}$ obtained in Lemma 5.1 and we consider the following initial value problem.

Problem $3 \mathcal{P}^{\beta_{\eta}}$. Find a bonding field $\beta_{\eta}:[0, T] \rightarrow L^{2}\left(\Gamma_{3}\right)$ such that

$$
\begin{aligned}
& \dot{\beta}_{\eta}(t)=-\left(\gamma_{v} \beta_{\eta}(t) R_{v}\left(u_{\eta v}(t)\right)^{2}-\varepsilon_{a}\right)_{+} \text {a.e. } t \in(0 T), \\
& \beta_{\eta}(0)=\beta_{0} .
\end{aligned}
$$

We obtain the following result.

Lemma 5.3 There exists a unique solution $\beta_{\eta}$ to Problem $\mathcal{P}^{\beta_{\eta}}$ and it satisfies $\beta_{\eta} \in$ $W^{1, \infty}\left(0, T, L^{2}\left(\Gamma_{3}\right)\right) \cap \mathcal{Q}$.

Proof Consider the mapping $F:[0, T] \times L^{2}\left(\Gamma_{3}\right) \rightarrow L^{2}\left(\Gamma_{3}\right)$ defined by

$$
F\left(t, \beta_{\eta}\right)=-\left(\gamma_{\nu} \beta_{\eta}(t) R_{v}\left(u_{\eta v}(t)\right)^{2}-\varepsilon_{a}\right)_{+},
$$

for all $t \in[0, T]$ and $\beta_{\eta} \in L^{2}\left(\Gamma_{3}\right)$. It follows from the properties of the truncation operator $R_{v}$ that $F$ is Lipschitz continuous with respect to the second argument, uniformly in time. Moreover, for any $\beta_{\eta} \in L^{2}\left(\Gamma_{3}\right)$, the mapping $t \mapsto F\left(t, \beta_{\eta}\right)$ belongs to $L^{\infty}\left(0, T ; L^{2}\left(\Gamma_{3}\right)\right)$. Using now a version of Cauchy-Lipschitz theorem (see, e.g., [27], p. 48), we obtain the existence of a unique function $\beta_{\eta} \in W^{1, \infty}\left(0, T, L^{2}\left(\Gamma_{3}\right)\right)$ which solves $(5.20),(5.21)$. We note that the restriction $0 \leq \beta_{\eta} \leq 1$ is implicitly included in the Cauchy problem $\mathcal{P}^{\beta_{\eta}}$. Indeed, (5.20) and (5.21) guarantee that $\beta_{\eta}(t) \leq \beta_{0}$, and therefore, assumption (3.9) shows that $\beta_{\eta}(t) \leq 1$ for $t \geq 0$, a.e. on $\Gamma_{3}$. On the other hand, if $\beta_{\eta}\left(t_{0}\right)=0$ at $t=t_{0}$, then it follows from (5.20) and (5.21) that $\dot{\beta}_{\eta}(t)=0$ for all $t \geq t_{0}$ and therefore, $\beta_{\eta}(t)=0$ for all $t \geq t_{0}$, a.e. on $\Gamma_{3}$. We conclude that $0 \leq \beta_{\eta}(t) \leq 1$ for all $t \in[0, T]$, a.e. on $\Gamma_{3}$. Therefore, from the definition of the set $\mathcal{Q}$, we find that $\beta_{\eta} \in \mathcal{Q}$, which concludes the proof of Lemma 5.3.

Now, for $\eta \in W^{1, \infty}(0, T ; V)$ we denote by $u_{\eta}$ and $\beta_{\eta}$ the functions obtained in Lemmata 5.1 and 5.3, respectively. We use Riesz's representation theorem to define the function $\Lambda \eta:[0, T] \longrightarrow V$ by

$$
(\Lambda \eta(t), v)_{V}=j\left(\beta_{\eta}(t), u_{\eta}(t), v\right)
$$

for all $v \in V$ and $t \in[0, T]$. We have the following result. 
Lemma 5.4 For all $\eta \in W^{1, \infty}(0, T ; V)$ the function $\Lambda \eta$ belongs to $W^{1, \infty}(0, T ; V)$. Moreover, there exists a unique element $\eta^{*} \in W^{1, \infty}(0, T ; V)$ such that

$$
\Lambda \eta^{*}=\eta^{*}
$$

Proof Let $\eta \in W^{1, \infty}(0, T ; V)$ and let $t_{1}, t_{2} \in[0, T]$. Using (5.23) and (3.19), we obtain

$$
\begin{aligned}
\| & \Lambda \eta\left(t_{1}\right)-\Lambda \eta\left(t_{2}\right)\left\|_{V} \leq c\right\| \beta_{\eta}^{2}\left(t_{1}\right) R_{v}\left(u_{\eta v}\left(t_{1}\right)\right) \\
& -\beta_{\eta}^{2}\left(t_{2}\right) R_{v}\left(u_{\eta v}\left(t_{2}\right)\right)\left\|_{L^{2}\left(\Gamma_{3}\right)}+c\right\| p_{\tau}\left(\beta_{\eta}\left(t_{1}\right)\right) R_{\tau}\left(u_{\eta \tau}\left(t_{1}\right)\right) \\
& -p_{\tau}\left(\beta_{\eta}\left(t_{2}\right)\right) R_{\tau}\left(u_{\eta \tau}\left(t_{2}\right)\right) \|_{L^{2}\left(\Gamma_{3}\right)} .
\end{aligned}
$$

Now, keeping in mind (3.5), (3.13), the inequality $0 \leq \beta_{\eta}(t) \leq 1$ and the properties of the truncation operators $R_{v}$ and $R_{\tau}$, we find that

$$
\begin{aligned}
\| & \Lambda \eta\left(t_{1}\right)-\Lambda \eta\left(t_{2}\right)\left\|_{V} \leq c\right\| u_{\eta}\left(t_{1}\right)-u_{\eta}\left(t_{2}\right) \|_{V} \\
& +c\left\|\beta_{\eta}\left(t_{1}\right)-\beta_{\eta}\left(t_{2}\right)\right\|_{L^{2}\left(\Gamma_{3}\right)} .
\end{aligned}
$$

Since $u_{\eta} \in W^{1, \infty}(0, T ; V)$ and $\beta_{\eta} \in W^{1, \infty}\left(0, T, L^{2}\left(\Gamma_{3}\right)\right) \cap \mathcal{Q}$, we deduce from inequality (5.25) that $\Lambda \eta \in W^{1, \infty}(0, T ; V)$.

Let now $\eta_{1}, \eta_{2} \in W^{1, \infty}(0, T ; V)$ and let $u_{i}=u_{\eta_{i}}, \dot{u}_{i}=\dot{u}_{\eta_{i}}, \beta_{i}=\beta_{\eta_{i}}$ for $i=1,2$. For $t \in[0, T]$ we integrate (5.20) with the initial conditions (5.21) to obtain

$$
\beta_{i}(t)=\beta_{0}-\int_{0}^{t}\left(\gamma_{\nu} \beta_{i}(s) R_{v}\left(u_{i v}(s)\right)^{2}-\varepsilon_{a}\right)_{+} d s .
$$

Using the definition of $R_{v}$, the inequality $\left|R_{v}\left(u_{v}\right)\right| \leq L$, and writing $\beta_{1}=\beta_{1}-\beta_{2}+$ $\beta_{2}$, we get

$$
\begin{aligned}
& \left\|\beta_{1}(t)-\beta_{2}(t)\right\|_{L^{2}\left(\Gamma_{3}\right)} \leq c \int_{0}^{t}\left\|\beta_{1}(s)-\beta_{2}(s)\right\|_{L^{2}\left(\Gamma_{3}\right)} d s \\
& +c \int_{0}^{t}\left\|u_{1 v}(s)-u_{2 v}(s)\right\|_{L^{2}\left(\Gamma_{3}\right)} d s .
\end{aligned}
$$

By Gronwall's inequality, it follows that

$$
\left\|\beta_{1}(t)-\beta_{2}(t)\right\|_{L^{2}\left(\Gamma_{3}\right)} \leq c \int_{0}^{t}\left\|u_{1 v}(s)-u_{2 v}(s)\right\|_{L^{2}\left(\Gamma_{3}\right)} d s,
$$


and, using (3.13) we obtain

$$
\left\|\beta_{1}(t)-\beta_{2}(t)\right\|_{L^{2}\left(\Gamma_{3}\right)} \leq c \int_{0}^{t}\left\|u_{1}(s)-u_{2}(s)\right\|_{V} d s
$$

On the other hand, using arguments similar to those in the proof of (5.25), we find that

$$
\left\|\Lambda \eta_{1}(t)-\Lambda \eta_{2}(t)\right\|_{V} \leq c\left\|u_{1}(t)-u_{2}(t)\right\|_{V}+c\left\|\beta_{1}(t)-\beta_{2}(t)\right\|_{L^{2}\left(\Gamma_{3}\right)}
$$

Then, by (5.26) we have

$$
\left\|\Lambda \eta_{1}(t)-\Lambda \eta_{2}(t)\right\|_{V} \leq c\left\|u_{1}(t)-u_{2}(t)\right\|_{V}+c \int_{0}^{t}\left\|u_{1}(s)-u_{2}(s)\right\|_{V} d s
$$

Next, we use (5.17) and (5.13) to find that

$$
\begin{aligned}
& \left(\dot{u}_{1}(t)-\dot{u}_{2}(t), u_{1}(t)-u_{2}(t)\right)_{V} \leq\left(\eta_{2}(t)-\eta_{1}(t), u_{1}(t)-u_{2}(t)\right)_{V} \\
& \quad+\left(\mathbf{G} u_{2}(t)-\mathbf{G} u_{1}(t), u_{1}(t)-u_{2}(t)\right)_{V},
\end{aligned}
$$

using Cauchy-Schwarz inequality and (5.12) we obtain

$$
\begin{aligned}
& \left(\dot{u}_{1}(t)-\dot{u}_{2}(t), u_{1}(t)-u_{2}(t)\right)_{V} \leq\left\|\eta_{1}(t)-\eta_{2}(t)\right\|_{V}\left\|u_{1}(t)-u_{2}(t)\right\|_{V} \\
& \quad+\left(\frac{L_{\mathcal{F}}}{m_{\mathcal{A}}}+\|L\|\right)\left\|u_{1}(t)-u_{2}(t)\right\|_{V}^{2} .
\end{aligned}
$$

We integrate this inequality with respect to time and use the initial conditions $u_{1}(0)=$ $u_{2}(0)=u_{0}$ to find that

$$
\begin{aligned}
& \frac{1}{2}\left\|u_{1}(t)-u_{2}(t)\right\|_{V}^{2} \leq \int_{0}^{t}\left\|\eta_{1}(s)-\eta_{2}(s)\right\|_{V}\left\|u_{1}(s)-u_{2}(s)\right\|_{V} d s \\
& \quad+\left(\frac{L_{\mathcal{F}}}{m_{\mathcal{A}}}+\|L\|\right) \int_{0}^{t}\left\|u_{1}(s)-u_{2}(s)\right\|_{V}^{2} d s .
\end{aligned}
$$

Applying the inequality

$$
a b \leq \frac{1}{2} a^{2}+\frac{1}{2} b^{2} a, b \in \mathbb{R}
$$

we find that

$$
\frac{1}{2}\left\|u_{1}(t)-u_{2}(t)\right\|_{V}^{2} \leq \frac{1}{2} \int_{0}^{t}\left\|\eta_{1}(s)-\eta_{2}(s)\right\|_{V}^{2} d s+\frac{1}{2} \int_{0}^{t}\left\|u_{1}(s)-u_{2}(s)\right\|_{V}^{2} d s
$$




$$
+\left(\frac{L_{\mathcal{F}}}{m_{\mathcal{A}}}+\|L\|\right) \int_{0}^{t}\left\|u_{1}(s)-u_{2}(s)\right\|_{V}^{2} d s
$$

where we obtain

$$
\left\|u_{1}(t)-u_{2}(t)\right\|_{V}^{2} \leq c \int_{0}^{t}\left\|\eta_{1}(s)-\eta_{2}(s)\right\|_{V}^{2} d s+c \int_{0}^{t}\left\|u_{1}(s)-u_{2}(s)\right\|_{V}^{2} d s,
$$

and, after a Gronwall argument, we obtain

$$
\left\|u_{1}(t)-u_{2}(t)\right\|_{V}^{2} \leq c \int_{0}^{t}\left\|\eta_{1}(s)-\eta_{2}(s)\right\|_{V}^{2} d s
$$

Using (5.27) we find that

$$
\begin{aligned}
& \left\|\Lambda \eta_{1}(t)-\Lambda \eta_{2}(t)\right\|_{V}^{2} \\
& \leq c^{2}\left\|u_{1}(t)-u_{2}(t)\right\|_{V}^{2}+c^{2}\left(\int_{0}^{t}\left\|u_{1}(s)-u_{2}(s)\right\|_{V} d s\right)^{2} \\
& +2 c\left\|u_{1}(t)-u_{2}(t)\right\|_{V} \cdot c \int_{0}^{t}\left\|u_{1}(s)-u_{2}(s)\right\|_{V} d s, \\
& \left\|\Lambda \eta_{1}(t)-\Lambda \eta_{2}(t)\right\|_{V}^{2} \\
& \leq c^{2}\left\|u_{1}(t)-u_{2}(t)\right\|_{V}^{2}+c^{2}\left(\int_{0}^{t}\left\|u_{1}(s)-u_{2}(s)\right\|_{V} d s\right)^{2} \\
& +c^{2}\left\|u_{1}(t)-u_{2}(t)\right\|_{V}^{2}+c^{2}\left(\int_{0}^{t}\left\|u_{1}(s)-u_{2}(s)\right\|_{V} d s\right)^{2}, \\
& \left\|\Lambda \eta_{1}(t)-\Lambda \eta_{2}(t)\right\|_{V}^{2} \\
& \leq 2 c^{2}\left\|u_{1}(t)-u_{2}(t)\right\|_{V}^{2}+2 c^{2}\left(\int_{0}^{t}\left\|u_{1}(s)-u_{2}(s)\right\|_{V} d s\right)^{2} .
\end{aligned}
$$

Using Cauchy-Schwarz inequality we find

$$
\left\|\Lambda \eta_{1}(t)-\Lambda \eta_{2}(t)\right\|_{V}^{2} \leq c\left\|u_{1}(t)-u_{2}(t)\right\|_{V}^{2}+c \int_{0}^{t}\left\|u_{1}(s)-u_{2}(s)\right\|_{V}^{2} d s
$$


We combine now (5.28) and (5.29) to see that

$$
\left\|\Lambda \eta_{1}(t)-\Lambda \eta_{2}(t)\right\|_{V}^{2} \leq c \int_{0}^{t}\left\|\eta_{1}(s)-\eta_{2}(s)\right\|_{V}^{2} d s \quad \forall t \in[0 T] .
$$

Reiterating this inequality $n$ times yields

$$
\left\|\Lambda^{n} \eta_{1}(t)-\Lambda^{n} \eta_{2}(t)\right\|_{L^{\infty}(0, T ; V)}^{2} \leq \frac{c^{n} T^{n}}{n !}\left\|\eta_{1}(t)-\eta_{2}(t)\right\|_{L^{\infty}(0, T ; V)}^{2}
$$

which implies that, for $n$ sufficiently large, a power $\Lambda^{n}$ of $\Lambda$ is a contraction in the Banach space $L^{\infty}(0, T ; V)$. Then, there exists a unique element $\eta^{*} \in L^{\infty}(0, T ; V)$ such that $\Lambda^{n} \eta^{*}=\eta^{*}$ and $\eta^{*}$ is also the unique fixed point of $\Lambda$, i.e., $\Lambda \eta^{*}=\eta^{*}$. The regularity $\eta^{*} \in W^{1, \infty}(0, T ; V)$ follows from the regularity $\Lambda \eta^{*} \in W^{1, \infty}(0, T ; V)$, which concludes the proof.

Now, we have all the ingredients necessary to prove Theorem 4.1.

Proof of Theorem 4.1 Existence. Let $\eta^{*} \in W^{1, \infty}(0, T ; V)$ be the fixed point of the operator $\Lambda$ and let $(u, \varphi, \beta)$ be the functions defined in Lemmata 5.1, 5.2, and 5.3, respectively, for $\eta=\eta^{*}$, i.e., $u=u_{\eta^{*}}, \varphi=\varphi_{\eta^{*}}, \beta=\beta_{\eta^{*}}$. Clearly, equalities (3.23), (3.24), and (3.26) hold from Lemmata 5.2 and 5.3. Moreover, since $\Lambda \eta^{*}=\eta^{*}$ it follows from (5.7), (5.5), (5.3), (5.8), and (5.23) that (3.22) and (3.25) hold, too. The regularity of the solution expressed in (4.1), (4.2), and (4.3) follows from Lemmata $5.1,5.2$, and 5.3, respectively.

Uniqueness. The uniqueness of the solution follows from the uniqueness of the fixed point of $\Lambda$ and the uniqueness part in Lemmata 5.1, 5.2, and 5.3.

Open Access This article is distributed under the terms of the Creative Commons Attribution 4.0 International License (http://creativecommons.org/licenses/by/4.0/), which permits unrestricted use, distribution, and reproduction in any medium, provided you give appropriate credit to the original author(s) and the source, provide a link to the Creative Commons license, and indicate if changes were made.

\section{References}

1. Bisenga, P., Lebon, F., Maceri, F.: The unilateral frictional contact of a piezoelectric body with a rigid support. In: Martins, J.A.C., Monteiro Marques, M.D.P. (eds.) Contact Mechanics, pp. 347-354. Kluwer, Dordrecht (2002)

2. Batra, R.C., Yang, J.S.: Saint-Venant's principle in linear piezoelectricity. J. Elast. 38, 209-218 (1995)

3. Barboteu, M., Sofonea, M.: Modeling and analysis of the unilateral contact of a piezoelectric body with a conductive support. J. Math. Anal. Appl. 358, 110-124 (2009)

4. Barboteu, M., Fernandez, J.R., Ouafik, Y.: Numerical analysis of two frictionless elastic-piezoelectric contact problems. J. Math. Anal. Appl. 339, 905-917 (2008)

5. Barboteu, M., Fernandez, J.R., Ouafik, Y.: Numerical analysis of a frictionless viscoelastic piezoelectric contact problem. Math. Model. Numer. Anal. 42(4), 667-682 (2008)

6. Barbu, V.: Optimal control of variational inequalities. Res. Notes Math. 100, 38-57 (1984) 
7. Chau, O., Fernández, J.R., Shillor, M., Sofonea, M.: Variational and numerical analysis of a quasistatic viscoelastic contact problem with adhesion. J. Comput. Appl. Math. 159, 431-465 (2003)

8. Chau, O., Shillor, M., Sofonea, M.: Dynamic frictionless contact with adhesion. J. Appl. Math. Phys. 55, 32-47 (2004)

9. Drabla, S., Zellagui, Z.: Variational analysis and the convergence of the finite element approximation of an electro-elastic contact problem with adhesion. Arab. J. Sci. Eng. 36, 1501-1515 (2011)

10. Frémond, M.: Equilibre des structures qui adhèrent à leur support. C. R. Acad. Sci. Paris Sér. II 295, 913-916 (1982)

11. Frémond, M.: Adhérence des solides. J. Méc. Théor. et Appl. 6, 383-407 (1987)

12. Frémond, M.: Non-Smooth Thermomechanics. Springer, Berlin (2002)

13. Han, W., Sofonea, M.: Quasistatic contact problems in viscoelasticity and viscoplasticity. In: Studies in Advanced Mathematics, vol. 30. American Mathematical Society, Providence, RI (2002)

14. Ikeda, T.: Fundamentals of Piezoelectricity. Oxford University Press, Oxford (1990)

15. Maceri, F., Bisegna, P.: The unilateral frictionless contact of a piezoelectric body with a rigid support. Math. Comput. Model. 28, 19-28 (1998)

16. Mindlin, R.D.: Polarisation gradient in elastic dielectrics. Int. J. Solids Struct. 4, 637-663 (1968)

17. Mindlin, R.D.: Continuum and lattice theories of influence of electromechanical coupling on capacitance of thin dielectric films. Int. J. Solids Struct. 4, 1197-1213 (1969)

18. Mindlin, R.D.: Elasticity, piezoelectricity and crystal lattice dynamics. J. Elast. 4, 217-280 (1972)

19. Morro, A., Straughan, B.: A uniqueness theorem in the dynamical theory of piezoelectricity. Math. Methods Appl. Sci. 14(5), 295-299 (1991)

20. Ouafik, Y.: Contribution à l'étude mathématique et num érique des structures piézoelectriques en Contact. Thèse, Université de Perpignan (2007)

21. Patron, V.Z., Kudryavtsev, B.A.: Electromagnetoelasticity, Piezoelectrics and Electrically Conductive Solids. Gordon \& Breach, London (1988)

22. Rojek, J., Telega, J.J.: Contact problems with friction, adhesion and wear in orthopaedic biomechanics. I: general developments. J. Theor. Appl. Mech. 39(3), 655-677 (2001)

23. Rojek, J., Telega, J.J., Stupkiewicz, S.: Contact problems with friction, adhesion and wear in orthopaedic biomechanics. II : numerical implementation and application to implanted knee joints. J. Theor. Appl. Mech. 39, 679-706 (2001)

24. Shillor, M., Sofonea, M., Telega, J.J.: Models and Variational Analysis of Quasistatic Contact. Lecture Notes in Physics, vol. 655. Springer, Berlin (2004)

25. Sofonea, M., Essoufi, El H.: A Piezoelectric contact problem with slip dependent coefficient of friction. Math. Model. Anal. 9, 229-242 (2004)

26. Sofonea, M., Essoufi, El H.: Quasistatic frictional contact of a viscoelastic piezoelectric body. Adv. Math. Sci. Appl. 14(1), 25-40 (2004)

27. Sofonea, M., Han, W., Shillor, M.: Analysis and Approximation of Contact Problems with Adhesion or Damage. Pure and Applied Mathematics, vol. 276. Chapman-Hall/CRC Press, New York (2006)

28. Sofonea, M., Ouafik, Y.: A piezoelectric contact problem with normal compliance. Appl. Math. 32, 425-442 (2005)

29. Toupin, R.A.: A dynamical theory of elastic dielectrics. Int. J. Eng. Sci. 1, 101-126 (1963)

30. Toupin, R.A.: Stress tensors in elastic dielectrics. Arch. Rational Mech. Anal. 5, 440-452 (1960)

31. Turbé, N., Maugin, G.A.: On the linear piezoelectricity of composite material. Math. Methods Appl. Sci. 14(6), 403-412 (1991) 